Article

\title{
The AaDREB1 Transcription Factor from the Cold-Tolerant Plant Adonis amurensis Enhances Abiotic Stress Tolerance in Transgenic Plant
}

\author{
Jun-Mei Zong ${ }^{\dagger}$, Xiao-Wei Li $^{\dagger}{ }^{,}$, Yuan-Hang Zhou, Fa-Wei Wang, Nan Wang, Yuan-Yuan Dong, \\ Yan-Xi Yuan, Huan Chen, Xiu-Ming Liu, Na Yao and Hai-Yan Li * \\ College of Life Sciences, Engineering Research Center of the Chinese Ministry of Education for Bioreactor and \\ Pharmaceutical Development, Jilin Agricultural University, Changchun 130118, Jilin, China; \\ zjm@frontier-ag.com (J.-M.Z.); xiaoweili1206@163.com (X.-W.L.); meyuanhang@163.com (Y.-H.Z.); \\ fw-1980@163.com (F.-W.W.); wangnanlunwen@126.com (N.W.); yydong@aliyun.com (Y.-Y.D.); \\ yuanyanxi123@163.com (Y.-X.Y.); chjlau@163.com (H.C.); xiuming1211@163.com (X.-M.L.); \\ yaona801103@aliyun.com (N.Y.) \\ * Correspondence: hyli99@163.com; Tel.: +86-431-8453-2885 \\ + These authors contributed equally to this work. \\ Academic Editor: Jianhua Zhu \\ Received: 26 November 2015; Accepted: 18 April 2016; Published: 22 April 2016
}

\begin{abstract}
Dehydration-responsive element binding (DREB) transcription factors (TFs) play important roles in the regulation of plant resistance to environmental stresses and can specifically bind to dehydration-responsive element/C-repeat element (DRE/CRT) proteins (G/ACCGAC) and activate expression of many stress-inducible genes. Here, we cloned and characterized a novel gene (AaDREB1) encoding the DREB1 transcription factor from the cold-tolerant plant Adonis amurensis. Quantitative real-time (qRT)-PCR results indicated that AaDREB1 expression was induced by salt, drought, cold stress, and abscisic acid application. A yeast one-hybrid assay demonstrated that $A a D R E B 1$ encodes a transcription activator and specifically binds to DRE/CRT. Furthermore, transgenic Arabidopsis and rice harboring AaDREB1 showed enhanced tolerance to salt, drought, and low temperature. These results indicated that AaDREB1 might be useful in genetic engineering to improve plant stress tolerance.
\end{abstract}

Keywords: AaDREB1; abiotic stress tolerance; Adonis amurensis; transgenic plant

\section{Introduction}

Plant growth and agricultural production are greatly constrained by environmental stresses, such as salinity, drought, extreme temperatures, UV irradiation and pathogen attacks. Plants, as sessile organisms, have evolved appropriate regulatory mechanisms that act at the cellular, molecular, physiological and biochemical levels to sense and rapidly adapt to stress conditions. Molecular and cellular responses to abiotic stresses involve signal perception, transduction of the signal to the cytoplasm and nucleus, alteration of gene expression and, finally, metabolic changes that lead to stress tolerance [1]. Among the stress-related genes, transcription factors (TFs) play an important role in regulating a plant's response to stress conditions. TFs act as master switches and trigger simultaneous expression of a large number of stress-response genes that contribute to the stress-tolerance phenotype.

All dehydration-responsive element binding (DREB) proteins belong to the DREB TFs subfamily of the APETALA2/ethylene-responsive element binding proteins (AP2/EREBP) family, contain a conserved DNA-binding domain, specifically bind the promoter regions of downstream genes, activate or suppress the transcription of these genes and finally enhance plant stress tolerance [2-4]. DREB homologous genes have been isolated from a variety of species [5], such as Arabidopsis [6], 
Zea mays [7], Gossypium hirsutum [8], rice [9], tomato [10], hot pepper [11], soybean [12], perennial ryegrass [13] and dwarf apple [14]. Expression of dehydration-responsive element binding1/C-repeat binding factor $(D R E B 1 / C B F)$ genes is strongly induced by cold, but not by dehydration and high-salt stresses, whereas expression of DREB2 genes is induced by dehydration and high-salt stresses but not by cold. These results suggest that two independent families of DRE-binding proteins function as trans-acting factors in two separate signal-transduction pathways under low-temperature and dehydration conditions, respectively [15-18]. Expression of CBF homologs can be induced by drought, high-salt and abscisic acid (ABA) treatment $[19,20]$. Overexpression of DREB1A/CBF3 under the control of the CaMV35S promoter also increased the tolerance to drought, high-salt, and freezing stresses $[15,21]$. Transgenic plants overexpressing either DREB1 or DREB2A genes enhanced tolerance to abiotic stress but also a marked decrease in plant height and delayed flowering time compared with wild-type plants [4,22-25].

Adonis amurensis is a cold-tolerant plant capable of sprouting and flowering prior to melting of ice and snow. The cold-responsive genes in this species therefore are of great research interest. Characterization of DREB genes from A. amurensis would contribute to our understanding of the molecular mechanisms of stress resistance to improve tolerance to adverse environments in economically important plants using transgenic technology. In this study, we cloned and characterized a novel gene (AaDREB1) from A. amurensis. Its expression pattern and transcription activation activity were investigated and overexpression of AaDREB1 in Arabidopsis thaliana and rice imparted enhanced tolerance to drought, salinity, and low temperature.

\section{Results}

\subsection{Isolation and Sequence Analysis of AaDREB1}

Sequence analysis confirmed isolation of a full-length cDNA of AaDREB1 (GenBank accession number: ADY68770.1) encoding a protein of 206 amino acids. The amino acids from 53 to 63 comprised a putative nuclear localization signal domain; from 68 to 131 they comprised a typical AP2 domain; and the amino acids between 136 and 273 contained a possible activation domain (Figure 1A). The deduced protein encoded by AaDREB1 also contained two conserved functional amino acids (valine and glutamic acid), located at the 14th and 19th residues in the domain and were thought to be crucial sites responsible for binding between DREB transcription factors and dehydration-responsive element (DRE) core sequences (TACCGACAT) [15] (Figure 1A). These features suggest that AaDREB1 encodes a possible AP2/EREBP transcription factor (TF). A BLAST analysis using NCBI databases also showed that $A a D R E B 1$ was homologous to DREB or DREB-like TFs from other plants. Cluster analysis of the deduced protein encoded by AaDREB1 showed that the AP2 domain of this protein had high homology with PtCBF6 (ABO48367; 48.84\%), PtDREB67 (XP-002328068; 47.27\%), and RcCBF (XP-002532187; $43.75 \%$ ) (Figure $1 \mathrm{~B})$.

\subsection{Expression Patterns of AaDREB1}

Expression patterns of $A a D R E B 1$ in response to the different stress treatments were analyzed (Figure 2). Under salt stress, expression of AaDREB1 began to increase after $1 \mathrm{~h}$, reached its maximum at $6 \mathrm{~h}$, and then gradually decreased after $12 \mathrm{~h}$ (Figure 2A). Similarly, expression was also induced by drought stress, mRNA accumulation peaked at $6 \mathrm{~h}$ and started to decline after $12 \mathrm{~h}$ (Figure 2B). Under low-temperature stress, transcription of $A a D R E B 1$ was strongly increased after $0.5 \mathrm{~h}$ and reached its maximum at $12 \mathrm{~h}$ (Figure 2C). In response to ABA treatment, AaDREB1 mRNA was weakly induced after $0.5 \mathrm{~h}$, peaked at $3 \mathrm{~h}$, and started to decline after $6 \mathrm{~h}$ (Figure 2D). Thus, $A a D R E B 1$ was induced by salt, drought, cold stresses, and ABA application. 
A
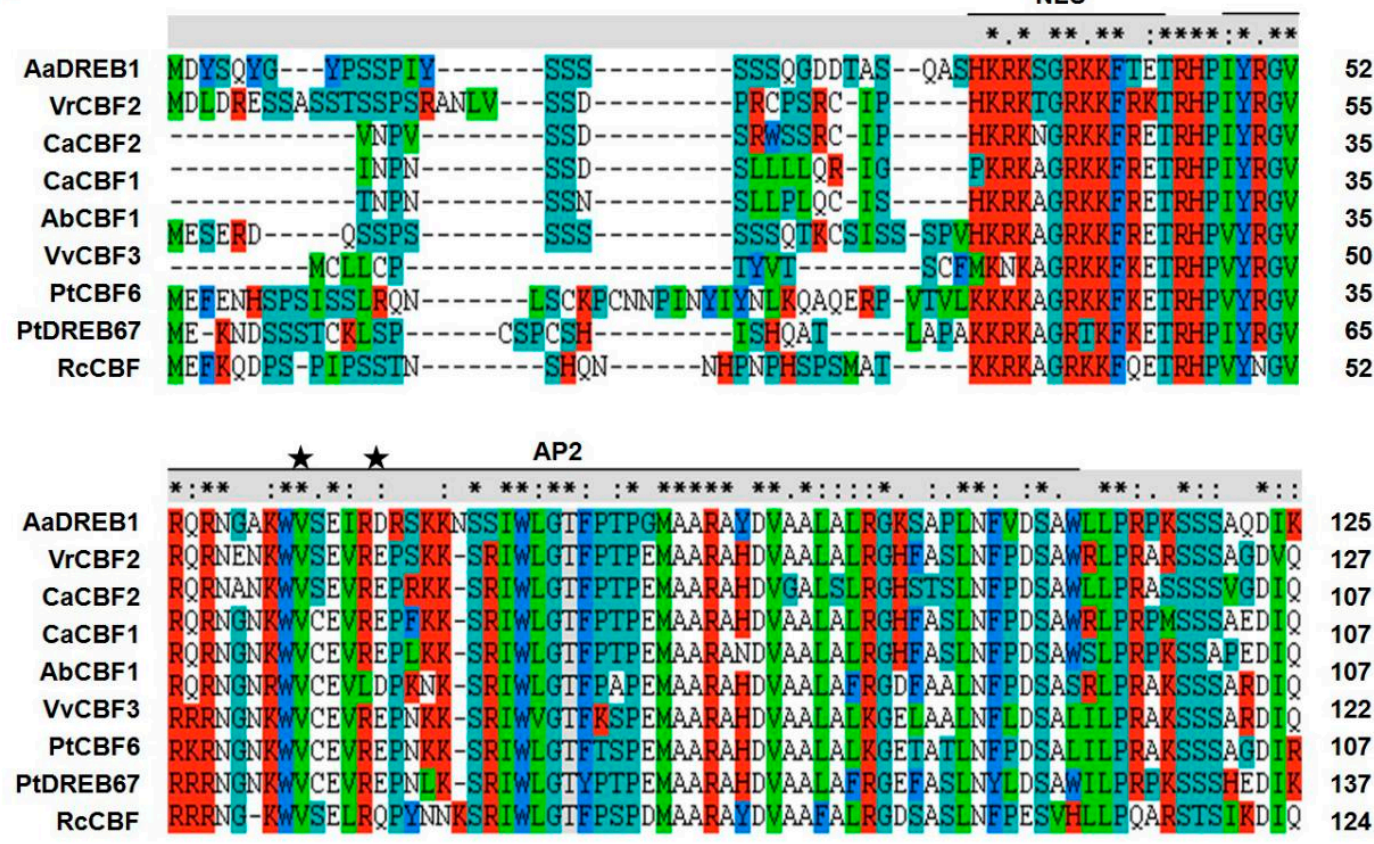

Activation domain

AaDREB1

VrCBF2

CaCBF2

CaCBF1

AbCBF1

VvCBF3

PtCBF6

PtDREB67

RcCBF

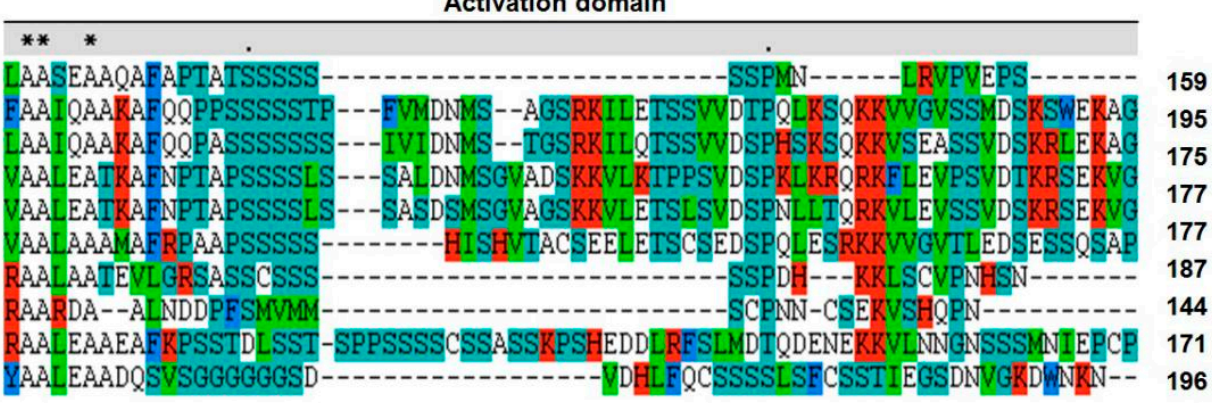

AaDREB1

$*^{* *}: *:^{* * * * *}::^{* * * *}::^{*}$

VrCBF2

CaCBF2

CaCBF1

AbCBF1

VvCBF3

PtCBF6

PtDREB67

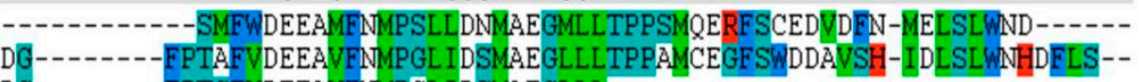

206

DG---- EPTGFVDEEAVFNMPCL IDSMAEGLLL

250

204

作

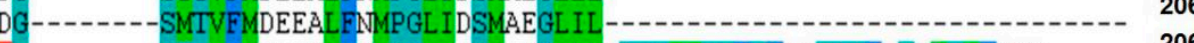

HG------SSTVFMDEEALFNMPGLINSMAEGLLLAPPTMLGGFSWDDTTSY-TDLSLMNDD----- 206

- ATFFDEEALFNMPGLLDSMEGL ILTPPAMARGVYWDDACS-TDLTLWEDDYLDSQ 239 -ALFFDEEALFNMPGLDSMAEGLILTPPVMQRGFN WDDMACS-TDLTLWEED---- 197

VSIEIRNECSVTSFLDDEALFNMPVLLDSMAEGLILTPPSIRRGFNWDDMAFA-VDLTLWRD----- $\mathbf{2 1 9}$

RcCBF -

Figure 1. Cont. 
B

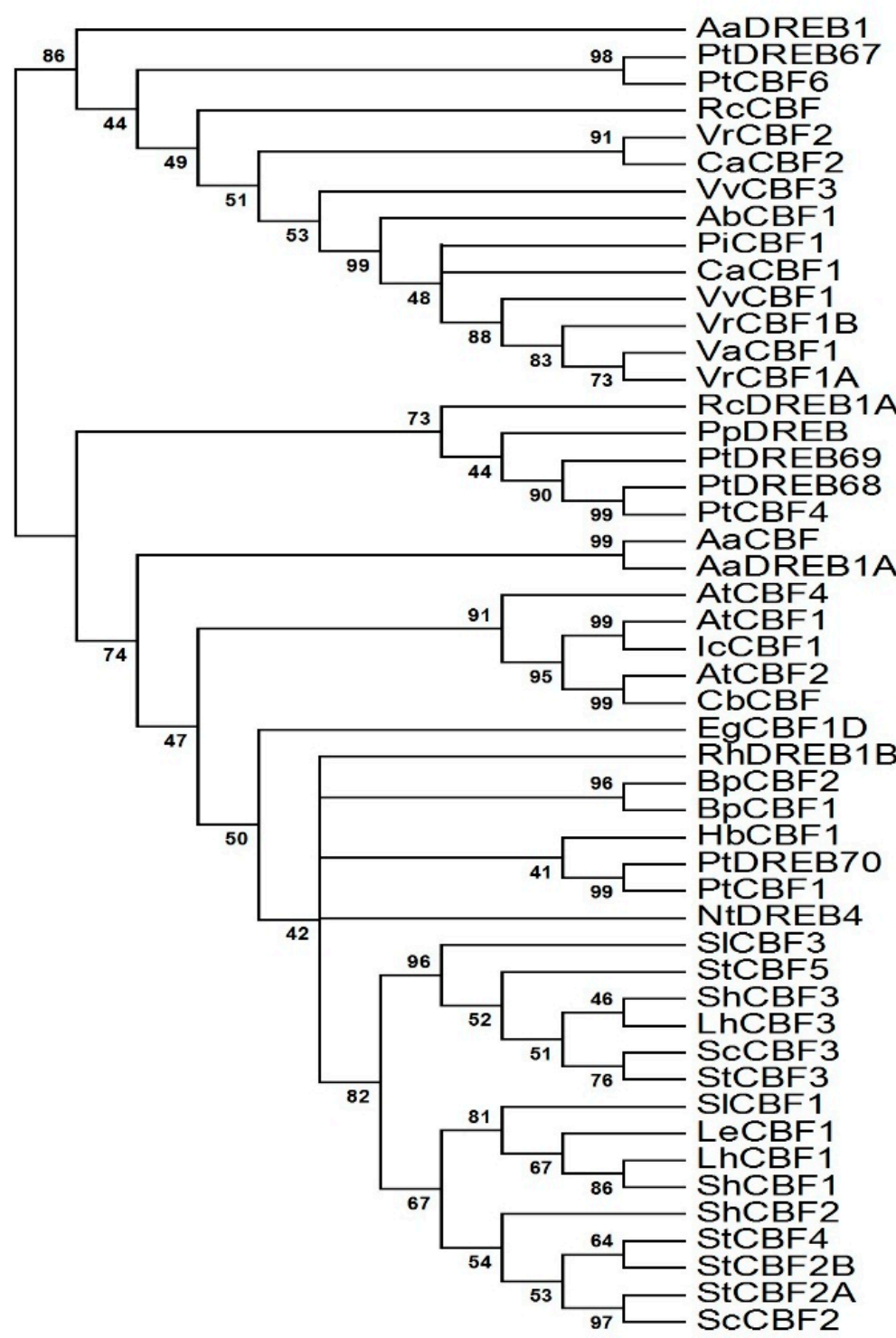

Figure 1. Comparison of the deduced amino acid sequence of Adonis amurensis dehydration-responsive element binding protein 1 (AaDREB1) and its homologs. Information of the DREB proteins of plants used here are in Supplementary Materials 1. (A) The sequences were aligned using CLUSTAL X. Gaps were introduced to optimize the alignment. The asterisks "** indicate positions with a single, fully conserved residue; ":" indicates highly conserved positions; and "." indicates more weakly conserved positions. Asterisks mark 14th and 19th amino acids of APETALA2/ethylene-responsive element-binding factor (AP2/ERF) domain. Dashes indicate gaps in the amino acid sequences. The column shows the score of the conservation positions. Lines above the alignment indicate the nuclear localization signal (NLS), the conserved AP2 domain, and the activation domain; identical and similar amino acids sequences are highlighted by red, deongaree, Cambridge blue and green shading respectively; (B) Phylogenetic analysis of Adonis amurensis and other plants' DREB family proteins. Analysis, based on minimum evolution, was performed with full-length protein sequences using the AP2 transcription factor as an outgroup. DREB proteins were initially aligned using Clustal W and were used for phylogenetic analysis using MEGA version 4.1 software. The phylogenetic tree was constructed using the neighbor-joining method with 1000 bootstrap replications. Bootstrap percentages are shown at dendrogram branch points. 

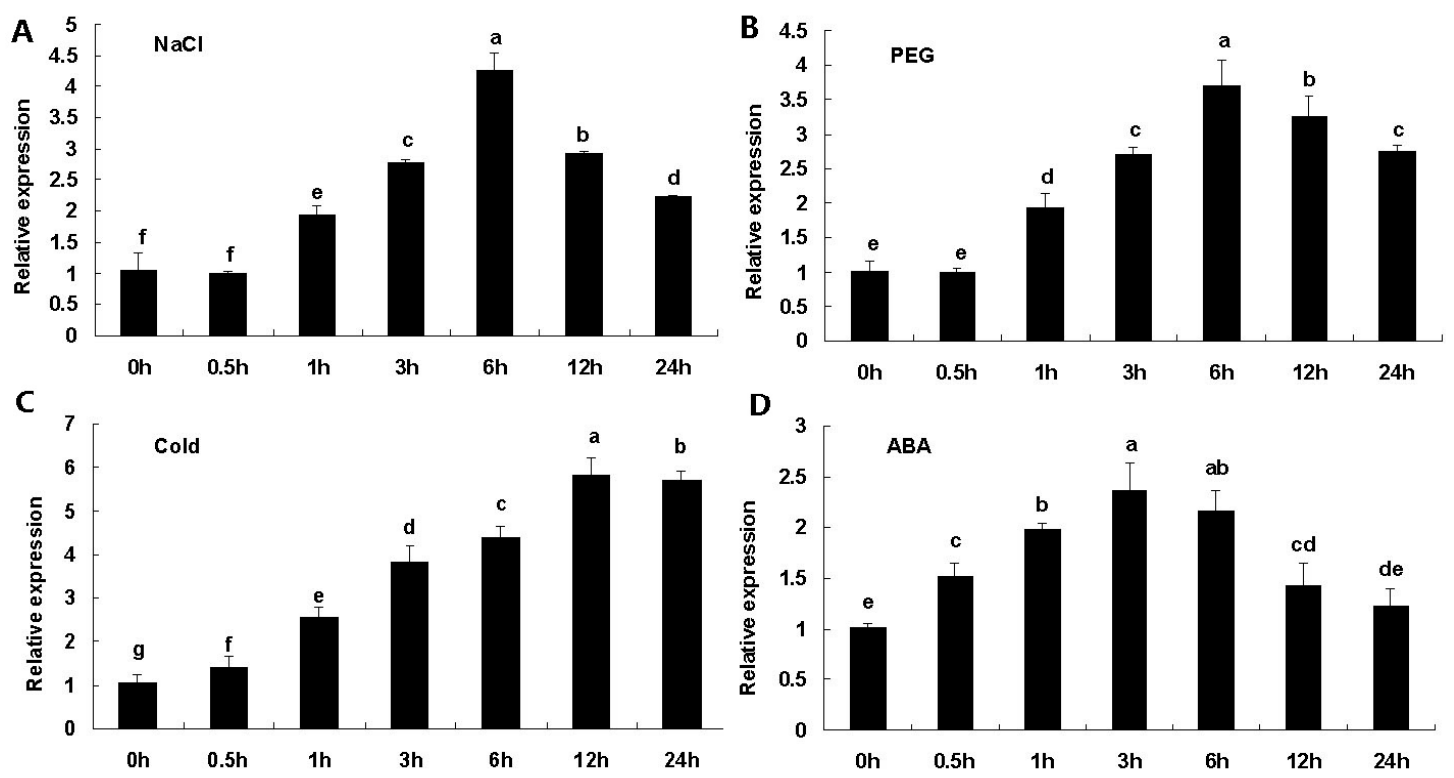

Figure 2. AaDREB1 expression patterns in response to different stress treatments. Leaves were collected at $0,0.5,1,3,6,12$ and $24 \mathrm{~h}$ after the initiation of high salinity (A), drought (B), low temperature (C) and abscisic acid (D). $\beta$-Actin gene was used as an internal standard. Values are means \pm standard errors of three biological repeats. Different letters on the column represent significant difference $(p<0.05)$ based on Duncan's multiple range test.

\subsection{Analysis of Trans-Activation Activity of AaDREB1}

All transformants grew normally on selective medium (SD/-Try) medium. The $\beta$-galactosidase activity assay showed that the pAD-AaDREB1 transformants of the dehydration-responsive element (DRE) yeast turned blue, while the mutant DRE (mDRE) transformants did not (Figure 3). Thus, heterogeneous expression of AaDREB1 promoted expression of the lacZ gene in wild-type DRE yeast but not in mutant DRE yeast, indicating that $A a D R E B 1$ encodes a transcription factor that can specifically bind to the DRE sequence in the promoter region and activate transcription of the downstream genes in vivo.

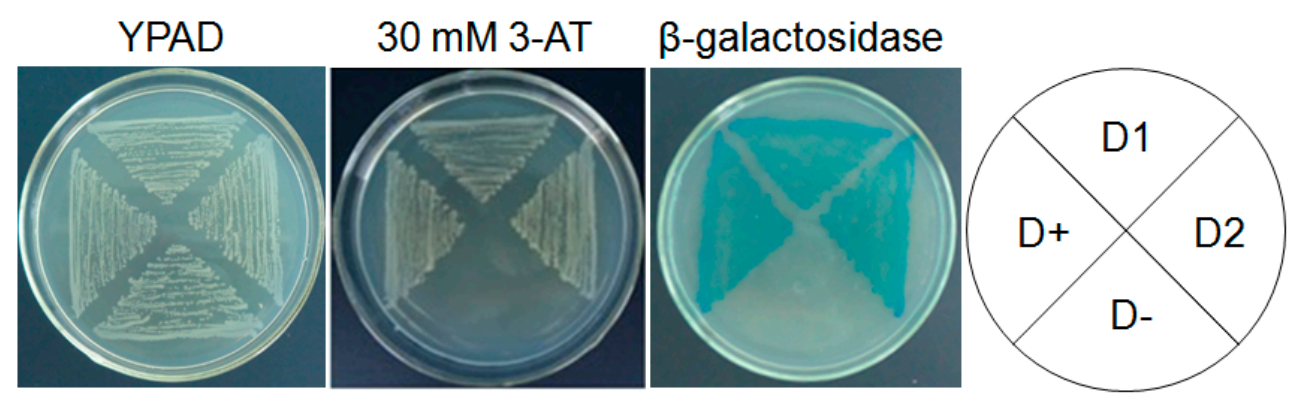

Figure 3. Yeast one-hybrid assays of AaDREB1. All yeast transformants carrying dual reporter genes were examined for growth in the presence of 3-AT, and $\beta$-galactosidase activity. The pAD-AaDREB1 was transformed into DRE yeast (D1, D2), with the DRE yeast containing the pAD-AtCBF3 as the positive control (D+) and the mDRE yeast containing the pAD-AtCBF3 as the negative control (D-). Transformants were streaked on YPAD medium or SD/-Try with $30 \mathrm{mM}$ 3-AT and grown at $30{ }^{\circ} \mathrm{C}$ for 2 days to observe growth and $\beta$-galactosidase activities. Left panel, YPAD medium; middle panel, $\mathrm{SD} /$-Try medium containing $30 \mathrm{mM} 3$-AT for growth analysis; right panel, $\beta$-galactosidase activity analysis results. 


\subsection{Overexpression of AaDREB1 Results in Enhanced Salt, Drought, and Cold Tolerance in Arabidopsis}

To examine the function of AaDREB1 in plant stress responses, the salt, drought, and cold tolerances of AaDREB1-overexpressing transgenic Arabidopsis lines and control plants were assessed. The wild-type plants withered and leaves were whitened, whereas the transgenic lines grew well with green leaves. Of the seedlings exposed to $-4{ }^{\circ} \mathrm{C}$ for $20 \mathrm{~h}$, the controls withered and died (Figure 4). These results indicated that overexpression of AaDREB1 in Arabidopsis greatly enhanced plant tolerance to salt, drought, and cold stresses, and that AaDREB1 might play important roles in plant stress signal transduction.

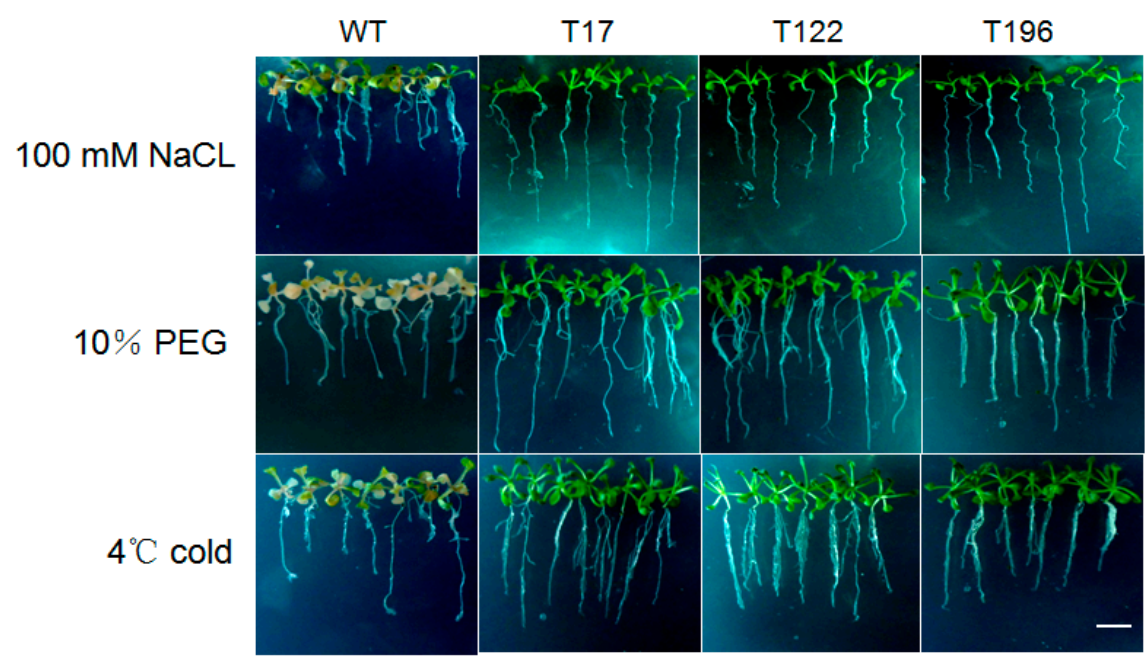

Figure 4. Stress-tolerance assays of AaDREB1 overexpressing transgenic Arabidopsis. Ten-day-old seedlings of AaDREB1 transgenic lines (T17, T122, T196) and the empty vector control plants (WT) were treated with either $100 \mathrm{mM} \mathrm{NaCl}$ for 12 days, $10 \%$ PEG for 10 days, or exposed to $4{ }^{\circ} \mathrm{C}$ for $20 \mathrm{~h}$, then grown under normal growth conditions for 3 days. Scale bar represents $1.5 \mathrm{~cm}$.

\subsection{Overexpression of AaDREB1 Improved Salt Tolerance of Transgenic Rice Plants}

The phenotypes and chlorophyll contents of AaDREB1-overexpressing transgenic rice were similar to those of wild-type rice plants grown under unstressed conditions (220-240 $\left.\mathrm{mg} \cdot \mathrm{g}^{-1} \mathrm{FW}\right)$ (Figure 5A,C). When plants were treated with $150 \mathrm{mM} \mathrm{NaCl}$, most wild-type plants withered and died (4.2\% survival rate, 4/94), whereas $25 \%(23 / 92), 37.3 \%(34 / 91)$, and $30.2 \%(29 / 96)$ of transgenic lines T4, T12, and T15 remained green and survived (Figure 5A,B). The average chlorophyll contents of transgenic lines (115-140 mg. $\mathrm{g}^{-1} \mathrm{FW}$ ) were significantly higher than that of the wild-type (15 mg. $\mathrm{g}^{-1} \mathrm{FW}$ ) (Figure 5C) in response to high salt treatment. These results indicated that overexpression of $A a D R E B 1$ enhanced salt tolerance of transgenic rice during the seedling stage.

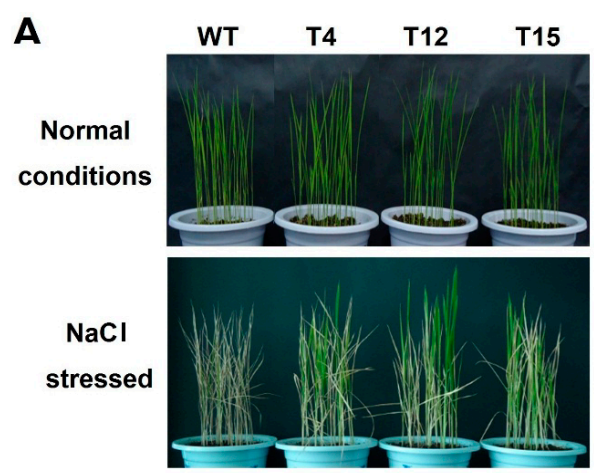

Figure 5. Cont. 

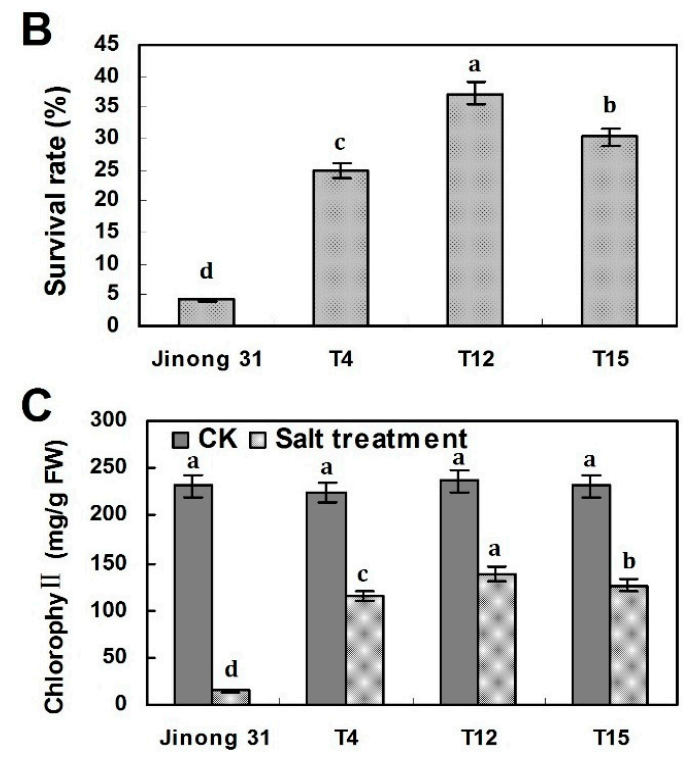

Figure 5. Salt-tolerance analyses of wild-type plants and AaDREB1 transgenic rice. (A) The phenotype of two-week-old AaDREB1 transgenic lines and wide-type rice plants grown in the glasshouse under normal growth conditions before being transferred to $150 \mathrm{mM} \mathrm{NaCl}$ for 16 days; (B) Survival rates of transgenic and wild-type plants after high-salt treatment; (C) The chlorophyll content of the leaves of transgenic and wild-type plants after high-salt treatment and under normal growth conditions. FW, fresh weight. Values are means \pm standard errors of three biological repeats. Different letters on the column represent significant difference $(p<0.05)$ based on Duncan's multiple range test.

\subsection{Overexpression of AaDREB1 Improved Drought Tolerance of Transgenic Rice Plants}

As shown in Figure 6A, seven-week-old $\mathrm{T}_{1}$ transgenic plants deprived of water for $12 \mathrm{~h}$ exhibited increased tolerance to drought stress, and the transgenic lines survived with parts of the leaves coiled, whereas the wild-type plants withered with stems bent and leaves coiled severely. Survival rates were 47.6\% (39/82) for 35S::AaDREB1 transgenic lines T4, and 44.3\% (43/97) and 45.1\% (41/91) for 35S::AaDREB1 transgenic lines T12 and T15, respectively, compared with 9.2\% (8/87) for wild-type plants (Figure 6B). To evaluate physiological changes in seven-week-old transgenic plants deprived of water for 15 days, soluble sugar contents in the leaves of different transgenic and wild-type plants were compared. Under control conditions, the soluble sugar contents in leaves were similar for different transgenic and wild-type plants (about $62 \mathrm{mg} \cdot \mathrm{g}^{-1} \mathrm{FW}$ ). However, following drought treatment, the soluble sugar levels in transgenic plants increased after 5 days, and reached $191-201 \mathrm{mg} \cdot \mathrm{g}^{-1} \mathrm{FW}$ at 15 days, whereas those in wild-type plants at the same developmental stage reached only $139 \mathrm{mg} \cdot \mathrm{g}^{-1}$ FW at 15 days. Statistical analysis showed that soluble sugar contents of transgenic rice plants were significantly higher than those of the wild-type at 5, 10, 15 and 20 days (Figure 6C).

\subsection{Overexpression of AaDREB1 Enhanced Freezing Tolerance of Transgenic Rice Plants}

$\mathrm{T}_{1}$ transgenic and wild-type plants were treated and allowed to recover prior to assessment of survival rates and free proline content analysis. After treatment with $4{ }^{\circ} \mathrm{C}$ for 12 days, wild-type plants showed retarded growth, whereas all transgenic lines grew well and only the leaf tips coiled slightly (Figure 7A). The survival rates of the transgenic lines were 54.5\%, (54/99) for T4, 62.9\% (61/97) for $\mathrm{T} 12$ and $58.7 \%$ (54/92) for T15, respectively, which were significantly higher than that of the wild-type plants $(7.3 \%, 7 / 96 ; p \leqslant 0.05)$ (Figure 7B). In addition, under normal growth conditions, free proline concentrations in the transgenic plants were approximately two-fold higher than the control plants (Figure 7C). This indicated that overexpression of AaDREB1 in rice improved freezing stress tolerance. 

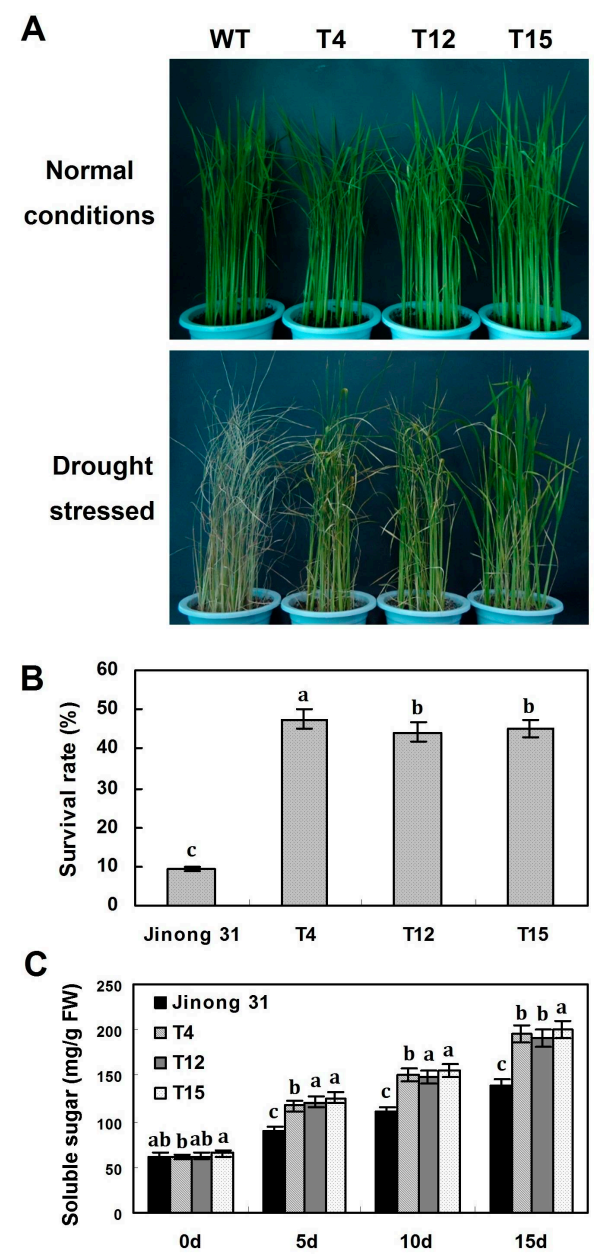

Figure 6. Drought tolerance analyses of wild-type plants and AaDREB1 transgenic rice. (A) The phenotype of transgenic and wild-type rice seedlings growing in soil in the glasshouse under normal growth conditions for seven weeks then were deprived of water for 15 days at $25{ }^{\circ} \mathrm{C}$; (B) Survival rate of AaDREB1 transgenic and wild-type plants after drought treatment; (C) Soluble sugar content of transgenic and wild-type plants. FW, fresh weight. Values are means \pm standard errors of three biological repeats. Different letters on the column represent significant difference $(p<0.05)$ based on Duncan's multiple range test.

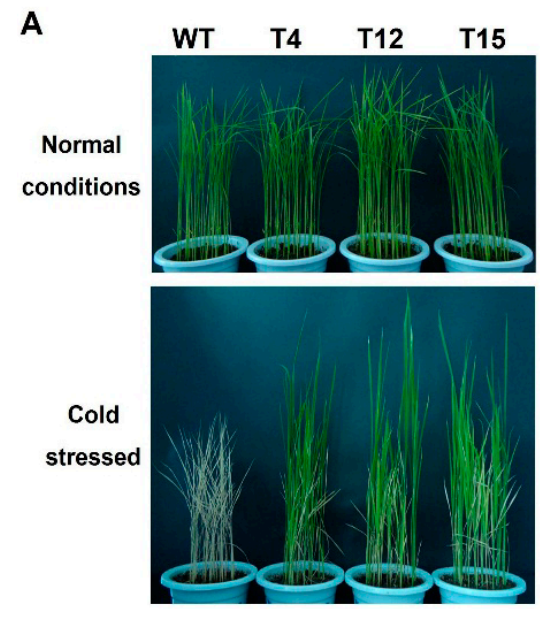

Figure 7. Cont. 
B
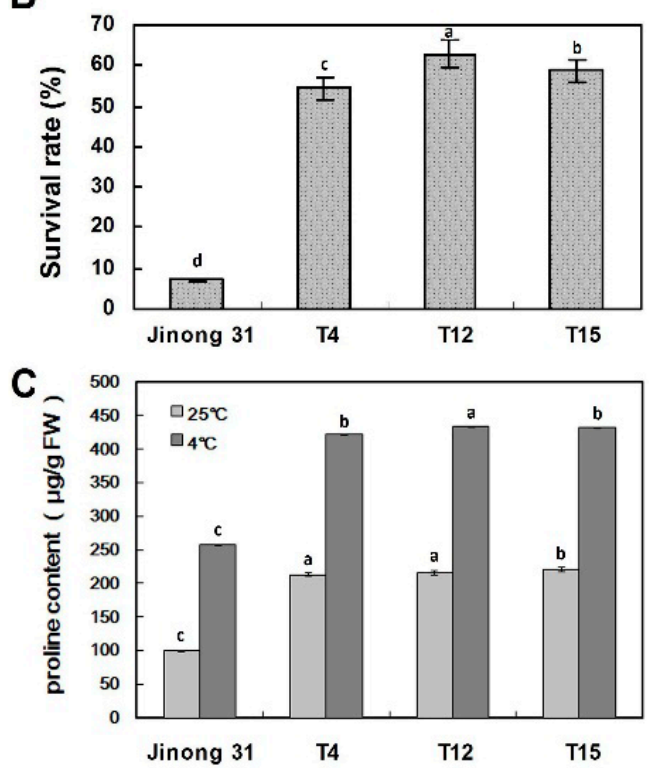

Figure 7. Low-temperature tolerance analyses of AaDREB1 transgenic and wild-type plants. (A) The phenotype of three-week-old AaDREB1 transgenic lines and wide-type rice plants grown in the glasshouse under normal growth conditions before being exposed to $4{ }^{\circ} \mathrm{C}$ for 12 days followed by recovery at $25^{\circ} \mathrm{C}$ for 5 days; (B) Survival rate of the control and three transgenic lines after cold treatment; (C) Proline concentration in three-week-old transgenic and wild-type rice plants after cold treatment. FW, fresh weight. Values are means \pm standard errors of three biological repeats. Different letters on the column represent significant difference $(p<0.05)$ based on Duncan's multiple range test.

\section{Discussion}

In this study, we isolated and characterized an AaDREB1 gene from the cold-tolerant plant $A$. amurensis. The deduced amino acid sequence of AaDREB1 contained a nuclear localization signal (NLS), an AP2 DNA-binding domain, and an activation domain flanking this domain, which was conserved in all of the other DREB1 transcription factors [2]. Sequence Blast analysis and classification revealed that AaDREB1 belongs to the DREB subfamily of the AP2/EREBP TF family and was placed in the A-1 group [26], which specifically binds to DRE/CRT elements and plays important roles in plant ABA-independent abiotic stress signaling pathways. The in vivo binding assay in yeast showed that AaDREB1 could specifically bind the DRE motif in the promoter and activate transcription of the fused reporter genes. Thus, AaDREB1 is a DRE-binding transcription factor in A. amurensis.

Expression of AaDREB1 in A. amurensis is induced by high salinity, drought, cold and ABA application. This pattern of expression is similar to that of OsDREB1F in rice [27], CBF1 in Arabidopsis [28], and ZmDREB2A in maize [7], but differs from that of OsDREB1A/B in rice [29], $Z m D R E B 1 A$ in maize [30], AtDREB1A/B/C in Arabidopsis [6,15] and GhDREB1 in cotton [31]. AaDREB1 responded to exogenous ABA, implying that it functions in an ABA-dependent pathway. The different expression patterns suggest that AaDREB1 may function differently from its homologs. The ABA-inducible characteristics suggested that AaDREB1 may also participate in ABA-dependent signal transduction pathways, which could be confirmed by examining the expression of downstream genes.

Overexpression of AaDREB1 in Arabidopsis and rice led to greatly enhanced tolerance to drought, high-salt and low-temperature conditions without growth retardation (Figures 4-7). These results indicated that overexpression of AaDREB1 may play important roles in plant stress signaling transduction. Overexpression of OsDREB1F in rice yielded similar results [28], while Ito et al. [32] 
reported that the overexpression of OsDREB1A, OsDREB1B, AtDREB1A, and AtDREB1B in rice resulted in various levels of growth inhibition under normal conditions. However, over-expression of AaDREB1 in Arabidopsis and rice had no impact on growth of plants. These results suggested that $A a D R E B 1$ probably functions differently from OsDREB1A and OsDREB1B, although all genes participated in stress signal transduction pathways.

Under drought, high-salt and low-temperature conditions, osmolytes such as various soluble sugars, in addition to free proline accumulate and play multiple roles in plant adaptation to stress [32,33]. Overexpression of GhDREB in transgenic wheat plants was associated with increased soluble sugar levels compared with wild-type plants under drought conditions [8], and overexpression of GhDREB1 in transgenic tobacco plants resulted in a higher level of free proline than in wild-type plants under low temperature conditions [31]. In our study, AaDREB1 transgenic plants maintained higher chlorophyll levels than control plants under high-salt stress (Figure 5C). A possible explanation was that overexpression of $A a D R E B 1$ activated expression of downstream genes that prevented chlorophyll decomposition, thus maintaining normal photosynthesis, and improving tolerance to high-salt stress [34]. Moreover, the AaDREB1 transgenic plants under normal conditions contained more soluble sugars than wild-type rice plants did, and the difference reached a significant level. $A a D R E B 1$ transgenic plants also accumulated higher levels of soluble sugars than wild-type plants under drought stress conditions (Figure 6C), suggesting that overexpression of AaDREB1 activated the expression of downstream genes involved in sugar biosynthesis, which in turn enhanced tolerance to drought stress in transgenic plants [12,32]. Under cold stress and normal growth temperatures, the free proline concentration in AaDREB1-overexpressing plants was approximately two-fold higher than that of control plants (Figure 7C). This might suggest a direct regulation of proline production processes by AaDREB1, and that AaDREB1 acts as a transcriptional activator in transgenic rice, which activates certain downstream genes involved in proline synthesis, conferring higher plant tolerance to cold stress and protecting the photosynthetic apparatus of plant cells through osmotic regulation [31]. Further research should be performed to study the mechanisms by which the AaDREB1 gene regulates plant tolerance to drought, salt and low-temperature stresses. However, the current results strongly suggest that $A a D R E B 1$ is an ideal candidate gene for the genetic manipulation of crops with the goal of abiotic stress-tolerance breeding.

\section{Experimental Section}

\subsection{Plant Materials and Stress Treatments}

Seedlings of $A$. amurensis were grown in pots $(13 \mathrm{~cm}$ diameter $\times 16 \mathrm{~cm}$ high) containing compost soil (leaf-mould:stable-litter:sand; 1:1:1) for 30 days, with a light/dark photoperiod of $16 / 8 \mathrm{~h}$ at $25^{\circ} \mathrm{C}$, prior to treatment. For the low-temperature treatment, uniformly developed seedlings were treated at $4{ }^{\circ} \mathrm{C}$. For other treatments, seedlings of $A$. amurensis were irrigated with $500 \mathrm{~mL}$ solutions containing $200 \mathrm{mM} \mathrm{NaCl}, 8 \%$ polyethylene glycol (PEG) 8000, or $100 \mu \mathrm{M}$ ABA, respectively. The leaves of the plants were harvested at $0,0.5,1,3,6,12$ and $24 \mathrm{~h}$ after treatment, directly put into liquid nitrogen and stored at $-80^{\circ} \mathrm{C}$ for qRT-PCR analysis. The experiment was repeated three times. Plant materials were sampled consistently at an appointed time after stress treatment for gene expression.

\subsection{Isolation of AaDREB1 from A. amurensis by RT-PCR and 5' and 3'RACE}

Bioinformatics methods and RT-PCR and 5' and $3^{\prime}$ rapid-amplification of cDNA ends (RACE) procedures were used to clone the full-length cDNA of the AaDREB1 protein from $A$. amurensis. To isolate the middle sequence of $A a D R E B 1$, degenerate primers were designed based on the conserved AP2 domain of the following dicotyledon DREB genes: AtDREB1b/AtCBF1 (At4g25490), AtDREB1c/AtCBF2 (At4g25470), AtDREB1a/AtCBF3 (At4g25480), AtDREB1d/AtCBF4 (At5g51990), AtDREB1F/DDF1 (At1g12610), AtDREB1E/DDF2 (At1g63030), ScCBF3 (ACB45092.1), RcDREB1A (XP_002509702.1), PpDREB (ABR19831.1), EgCBF1D (ACF15447.1), 
NtDREB4 (ACE73696.1), RhDREB1B (ACI42860.1) and PtDREB69 (XP_002298067.1). The multiple alignment of these genes above is in Figure S1. The primer sequences are as follows: forward primer, 5'-TTYMRDGAGACDMGDCACCC-3'; reverse primer, 5'-ARRAGMADNCCYTCNGCCAT3'. In the sequences, $\mathrm{Y}$ denotes $\mathrm{C}$ or $\mathrm{T}, \mathrm{M}$ denotes $\mathrm{A}$ or $\mathrm{C}, \mathrm{R}$ denotes $\mathrm{A}$ or $\mathrm{G}, \mathrm{D}$ denotes $\mathrm{A}, \mathrm{G}$ or $\mathrm{T}$, and $\mathrm{N}$ denotes any nucleotide. Total RNA was extracted from leaves of $A$. amurensis that were treated at $4{ }^{\circ} \mathrm{C}$ for $2 \mathrm{~h}$ using TRIzol ${ }^{\circledR}$ reagent (Invitrogen, Carlsbad, CA, USA) and then treated with RNase-free DNase (Invitrogen). One microgram of treated RNA was used for first-strand cDNA synthesis and PCR. The annealing temperature in PCRs was $54^{\circ} \mathrm{C}$. The PCR products were cloned into the pGEM ${ }^{\circledR}$-T Easy Vector (Promega, New York, NY, USA) and sequenced. Specific primers were designed for rapid amplification of the cDNA end according to the sequence information of the partial cDNA fragment to obtain the full-length sequence of the gene. For $5^{\prime}$ RACE, two antisense gene-specific primers (GSP) were designed: GSP1F (5'-ATCTTCGTCGTCGTCTTCCC-3') and GSP2F (5'-TTCCCTCTGAGTGCCAAAGC-3'). Primers for $3^{\prime}$ RACE were: GSP1R (5'-TTCTGGGATGAGGAGGCAAT-3') and GSP2R (5'-GGGAATGTGCCTAGCCATAT-3'). The RACE reactions were performed with the GeneRacer ${ }^{\mathrm{TM}}$ kit (Invitrogen). The RACE products were cloned and sequenced. A full-length cDNA sequence was obtained by combining the 5' RACE, cDNA and 3' RACE fragments. The full-length cDNA sequence of AaDREB1 was amplified by PCR using the primers GSP3F (5'-ATGGATTATTCGCAGTACGG-3') and GSP3R (5'-TTAATCGTTCCACAAGGACA-3').

\subsection{Quantitative Real-Time PCR Analysis}

Total RNA was extracted from plants using the RNA Plant Plus Reagent (Tiangen, Changchun, China) according to the manufacturer protocol and cDNA was reverse transcribed using M-MLV reverse transcriptase (TaKaRa, Changchun, China). Quantitative real-time PCR analysis was performed using SYBR Green I dye (TaKaRa, Changchun, China) and a real-time PCR machine (Applied Biosystems 7500, Foster City, CA, USA). The qRT-PCR cycling stages consisted of initial denaturation at $95{ }^{\circ} \mathrm{C}(30 \mathrm{~s})$, followed by 40 cycles of $95{ }^{\circ} \mathrm{C}(5 \mathrm{~s}), 58{ }^{\circ} \mathrm{C}(34 \mathrm{~s})$, and a final melting curve stage of $95{ }^{\circ} \mathrm{C}(15 \mathrm{~s}), 60^{\circ} \mathrm{C}(1 \mathrm{~min})$, and $95{ }^{\circ} \mathrm{C}(15 \mathrm{~s})$. The fluorescence signal was recorded during the strand elongation step at $58{ }^{\circ} \mathrm{C}$ and the melting curve stage at every $0.3{ }^{\circ} \mathrm{C}$ temperature ramp. Samples for qRT-PCR were run in three biological replicates and three technical replicates. Relative gene expression was calculated using the comparative DDCT method according to Livak and Schmittgen [35]. The $\beta$-actin gene was chosen as an internal control, specific primers used here were Actin-F: $5^{\prime}$-ACTGTGCCAATCTACGAGGG-3' and Actin-R: 5'-TCTTACAATTTCCCGCTCTG-3'. Specific primers AaDREB1-qF: 5'-TATCAAACTCGCTGCTTCT-3' and AaDREB1-qR: 5'-TCATCCCAGAACATAGAAG-3' were used to amplify the AaDREB1 gene. The experiment was repeated three times, and the results from three samples were averaged.

\subsection{Comparison of the Deduced Amino Acid Sequence of Adonis Amurensis AaDREB1 and Its Homologs}

The sequences were aligned using ClustalX. DREB proteins were initially aligned using ClustalW and were used for phylogenetic analysis using MEGA version 4.1. The phylogenetic tree was constructed using the neighbor-joining method with 1000 bootstrap replications. Information regarding the DREB proteins of plants used here are in Supplementary Materials 1.

\subsection{In Vivo Dehydration-Responsive Element (DRE)-Binding and Transactivation Activity Using a Yeast One-Hybrid System}

To analyze DRE-binding activity and transactivation activity of the isolated cDNA clone, the full-length cDNA insert was cloned into the yeast expression vector pAD-GAL4-2.1, and this plasmid was transformed into DRE and mDRE strains following [25]. The DRE yeast containing pAD-AtCBF3 was the positive control (+), and the mDRE yeast containing pAD-AtCBF3 was the negative control $(-)$. The transformants were incubated on yeast extract-peptone-dextrose (YPAD) medium for 2 days to examine their growth and assay $\beta$-galactosidase activity. 


\subsection{Construction of the Plant Overexpression Vector and Transformation}

The AaDREB1 gene, amplified using the primers 5'-CGGGATCCATGGATTATTCGCAGTACGG-3' and 5'-CGAGCTCTTAATCGTTCCACAAGGACA-3', was digested with BamH I (GGATCC) and Sac I (GAGCTC), and the entire AaDREB1 coding region was cloned into the BamH I and Sac I sites of the binary vector $\mathrm{pBI} 121$ under the control of the CaMV35S promoter in the sense orientation. The plant overexpression vector for AaDREB1, PBI121-AaDREB1, was introduced into Agrobacterium tumefaciens strain EHA105. Arabidopsis thaliana and rice were used for transformation. Transformed plants carrying PBI121-AaDREB1 were identified by PCR. $\mathrm{T}_{1}$ generation plants were used in all experiments.

\subsection{Analyses of Stress Tolerance and Physiological Changes in Transgenic Arabidopsis and Rice}

Three lines (T17, T122 and T196) of transgenic $\mathrm{T}_{3}$ Arabidopsis plants were selected from among the transformants overexpressing $A a D R E B 1$ to assess tolerance to cold, drought, or salt. Seeds of both wild-type and transgenic lines were germinated on Murashige and Skoog (MS) agar medium for 10 days. The seedlings were transferred to MS medium supplemented with either $100 \mathrm{mM} \mathrm{NaCl}$ for 12 days to assess salt tolerance, or 10\% PEG for 10 days to assess drought tolerance. To evaluate cold tolerance, 14-day-old seedlings were exposed to $4{ }^{\circ} \mathrm{C}$ for $20 \mathrm{~h}$ and then grown under normal conditions for 3 days.

Wild-type rice ( $c v$. Jinong31) and transgenic rice seeds were grown in pots $(13 \mathrm{~cm}$ diameter $\times 16 \mathrm{~cm}$ high) at $28^{\circ} \mathrm{C}$ under continuous illumination. Two-week-old seedlings were used for all treatments. For salt tolerance analyses, wild-type and transgenic seedlings were irrigated with $1000 \mathrm{~mL}$ at two-day intervals with $150 \mathrm{mM} \mathrm{NaCl}$ for 16 days and then with water for 15 days before measurement of survival rate and chlorophyll content. The experiment was repeated three times and 25-30 plants were analyzed in each experiment. Chlorophyll content was determined following Hardwick and Baker [36]. To further examine drought tolerance and physiological changes, wild-type and transgenic seedlings were deprived of water, and leaves were collected after 0,5, 10, and 15 days for measurement of the soluble sugar content. After drought stress for 15 days, plants were rewatered for analysis of survival rate and soluble sugar content measured as described previously by Taji et al. [37]. For freezing tolerance analyses, wild-type and transgenic seedlings were treated with low temperature $\left(4{ }^{\circ} \mathrm{C}\right)$ for 12 days, followed by transfer to a greenhouse for recovery. After 10 days, the survival rate and proline content were analyzed following Bates et al. [38].

\subsection{Statistical Analysis}

Data analysis was carried out using SPSS 13.0 (SPSS, Chicago, IL, USA). The means were tested using one-way analysis of variance (ANOVA). Duncan's tests were used for multiple comparisons between treatments. All data were represented by an average of three replicate measurements and standard error. The significance level was $p<0.05$.

\section{Conclusions}

In this work, we cloned and characterized a novel gene (AaDREB1) encoding the DREB1 transcription factor from $A$. amurensis, which is a cold-tolerant plant capable of sprouting and flowering prior to melting of ice and snow. Quantitative RT-PCR results indicated that AaDREB1 expression was induced by salt, drought, cold stress, and abscisic acid application. A yeast one-hybrid assay demonstrated that AaDREB1 encodes a transcription activator and specifically binds to DRE/CRT. Furthermore, AaDREB1 transgenic rice showed improved salt, drought and cold tolerance but displayed no marked decrease in plant height compared with wild-type plants.

Supplementary Materials: Supplementary materials can be found at http:/ /www.mdpi.com/1422-0067/17/ 4/611/s1. 
Acknowledgments: This research was supported by the Special Program for Research of Transgenic Plants (2014ZX08010-002) and the National Natural Science Foundation of China (31271746, 31201144, 31401403, 31101091).

Author Contributions: Hai-Yan Li conceived and designed the experiments; Jun-Mei Zong, Yuan-Hang Zhou, Fa-Wei Wang and Nan Wang performed the experiments; Xiao-Wei Li, Yuan-Yuan Dong and Yan-Xi Yuan analyzed the data; Huan Chen, Xiu-Ming Liu and Na Yao checked the manuscript; Xiao-Wei Li and Jun-Mei Zong wrote the paper; Hai-Yan Li read and approved the final manuscript; All authors have read and approved the final manuscript.

Conflicts of Interest: The authors declare no conflict of interest.

\section{References}

1. Agarwal, P.K.; Agarwal, P.; Reddy, M.K.; Sopory, S.K. Role of DREB transcription factors in abiotic and biotic stress tolerance in plants. Plant Cell Rep. 2006, 25, 1263-1274. [CrossRef] [PubMed]

2. Okamuro, J.K.; Caster, B.; Villarroel, R.; van Montagu, M.; Jofuku, K.D. The AP2 domain of APETALA2 defines a large new family of DNA binding proteins in Arabidopsis. Proc. Natl. Acad. Sci. USA 1997, 94, 7076-7081. [CrossRef] [PubMed]

3. Feng, J.X.; Liu, D.; Pan, Y.; Gong, W.; Ma, L.G.; Luo, J.C.; Deng, X.W.; Zhu, Y.X. An annotation update via cDNA sequence analysis and comprehensive profiling of developmental, hormonal or environmental responsiveness of the Arabidopsis AP2/EREBP transcription factor gene family. Plant Mol. Biol. 2005, 59, 853-868. [CrossRef] [PubMed]

4. Matsukura, S.; Mizoi, J.; Yoshida, T.; Todaka, D.; Ito, Y.; Maruyama, K.; Shinozaki, K.; Yamaguchi-Shinozaki, K. Comprehensive analysis of rice DREB2-type genes that encode transcription factors involved in the expression of abiotic stress-responsive genes. Mol. Genet. Genom. 2010, 283, 185-196. [CrossRef] [PubMed]

5. Lata, C.; Prasad, M. Role of DREBs in regulation of abiotic stress responses in plants. J. Exp. Bot. 2011, 62, 4731-4748. [CrossRef] [PubMed]

6. Novillo, F.; Medina, J.; Salinas, J. Arabidopsis CBF1 and CBF3 have a different function than CBF2 in cold acclimation and define different gene classes in the CBF regulon. Proc. Natl. Acad. Sci. USA 2007, 104, 21002-21007. [CrossRef] [PubMed]

7. Nguyen, H.T.; Leipner, J.; Stamp, P.; Guerra-Peraza, O. Low temperature stress in maize (Zea may L.) induces genes involved in photosynthesis and signal transduction as studied by suppression subtractive hybridization. Plant Physiol. Biochem. 2008, 47, 116-122. [CrossRef] [PubMed]

8. Gao, S.Q.; Chen, M.; Xia, L.Q.; Xiu, H.J.; Xu, Z.H.; Li, L.C.; Zhao, C.P.; Cheng, X.G.; Ma, Y.Z. A cotton (Gossypium hirsutum) DRE-binding transcription factor gene, GhDREB, confers enhanced tolerance to drought, high salt, and freezing stresses in transgenic wheat. Plant Cell Rep. 2009, 28, 301-311. [CrossRef] [PubMed]

9. Tian, X.H.; Li, X.P.; Zhou, H.L.; Zhang, J.S.; Gong, Z.Z.; Chen, S.Y. OsDREB4 genes in rice encode AP2-containing proteins that bind specifically to the dehydration-responsive element. Acta Bot. Sin. 2005, 47, 467-476. [CrossRef]

10. Gutterson, N.; Reuber, T.L. Regulation of disease resistance pathways by AP2/ERF transcription factors. Curr. Opin. Plant Biol. 2004, 7, 465-471. [CrossRef] [PubMed]

11. Hong, J.P.; Kim, W.T. Isolation and functional characterization of the CaDREBLP1 gene encoding a dehydration-responsive element binding-factor-like protein 1 in hot pepper (Capsicum annuum L. cv. Pukang). Planta 2005, 220, 875-888. [CrossRef] [PubMed]

12. Chen, M.; Wang, Q.Y.; Cheng, X.G.; Xu, Z.S.; Li, L.C.; Ye, X.G.; Xia, L.Q.; Ma, Y.Z. GmDREB2, a soybean DRE-binding transcription factor, conferred drought and high-salt tolerance in transgenic plants. Biochem. Biophys. Res. Commun. 2007, 353, 299-305. [CrossRef] [PubMed]

13. Xiong, Y.W.; Fei, S.Z. Function and phylogenenetic analysis of a DREB/CBF-like gene in perennial ryegrass (Lolium perenne L.). Planta 2006, 224, 878-888. [CrossRef] [PubMed]

14. Yang, W.; Liu, X.D.; Chi, X.J.; Wu, C.A.; Li, Y.Z.; Song, L.L.; Liu, X.M.; Wang, Y.F.; Wang, F.W.; Zhang, C.; et al. Dwarf apple MbDREB1 enhances plant tolerance to low temperature, drought, and salt stress via both ABA-dependent and ABA-independent pathway. Planta 2011, 233, 219-229. [CrossRef] [PubMed] 
15. Liu, Q.; Kasuga, M.; Sakuma, Y.; Abe, H.; Miura, S.; Yamaguchi-Shinozaki, K.; Shinozaki, K. Two transcription factors, DREB1 and DREB2, with an EREBP/AP2 DNA binding domain separate two cellular signal transduction pathways in drought- and low-temperature-responsive gene expression, respectively, in Arabidopsis. Plant Cell 1998, 10, 1391-1406. [CrossRef] [PubMed]

16. Shinwari, Z.K.; Nakashima, K.; Miura, S.; Kasuga, M.; Seki, M.; Yamaguchi-Shinozaki, K.; Shinozaki, K. An Arabidopsis gene family encoding DRE/CRT binding proteins involved in low-temperature-responsive gene expression. Biochem. Biophys. Res. Commun. 1998, 250, 161-170. [CrossRef] [PubMed]

17. Shinozaki, K.; Yamaguchi-Shinozaki, K.; Seki, M. Regulatory network of gene expression in the drought and cold stress responses. Curr. Opin. Plant Biol. 2003, 6, 410-417. [CrossRef]

18. Sakuma, Y.; Maruyama, K.; Osakabe, Y.; Qin, F.; Seki, M.; Shinozaki, K.; Yamaguchi-Shinozaki, K. Functional analysis of an Arabidopsis transcription factor, DREB2A, involved in drought-responsive gene expression. Plant Cell 2006, 18, 1292-1309. [CrossRef] [PubMed]

19. Haake, V.; Cook, D.; Riechmann, J.L.; Pineda, O.; Thomashow, M.F.; Zhang, J.Z. Transcription factor CBF4 is a regulator of drought adaptation in Arabidopsis. Plant Physiol. 2002, 130, 639-648. [CrossRef] [PubMed]

20. Knight, H.; Zarka, D.G.; Okamoto, H.; Thomashow, M.F.; Knight, M.R. Abscisic acid induces CBF gene transcription and subsequent induction of cold-regulated genes via the CRT promoter element. Plant Physiol. 2004, 135, 1710-1717. [CrossRef] [PubMed]

21. Kasuga, M.; Liu, Q.; Miura, S.; Yamaguchi-Shinozaki, K.; Shinozaki, K. Improving plant drought, salt, and freezing tolerance by gene transfer of a single stress-inducible transcription factor. Nat. Biotechnol. 1999, 17, 287-291. [PubMed]

22. Kasuga, M.; Miura, S.; Shinozaki, K.; Yamaguchi-Shinozaki, K. A combination of the Arabidopsis DREB1A gene and stress-inducible $r d 29 A$ promoter improved drought- and low-temperature stress tolerance in tobacco by gene transfer. Plant Cell Physiol. 2004, 45, 346-350. [CrossRef] [PubMed]

23. Fang, Z.W.; Zhang, X.H.; Gao, J.F.; Wang, P.K.; Xu, X.Y.; Liu, Z.X.; Shen, S.H.; Feng, B.L. A buckwheat (Fagopyrum esculentum) DRE-binding transcription factor gene, FeDREB1, enhances freezing and drought tolerance of transgenic Arabidopsis. Plant Mol. Biol. Rep. 2015, 33, 1-16. [CrossRef]

24. Sadhukhan, A.; Kobayashi, Y.; Kobayashi, Y.; Tokizawa, M.; Yamamoto, Y.Y.; Iuchi, S.; Koyama, H.; Panda, S.K.; Sahoo, L. VuDREB2A, a novel DREB2-type transcription factor in the drought-tolerant legume cowpea, mediates DRE- dependent expression of stress responsive genes and confers enhanced drought resistance in transgenic Arabidopsis. Planta 2014, 240, 645-664. [CrossRef] [PubMed]

25. Bouaziz, D.; Pirrello, J.; Charfeddine, M.; Hammami, A.; Jbir, R.; Dhieb, A.; Bouzayen, M.; Gargouri-Bouzid, R. Overexpression of StDREB1 transcription factor increases tolerance to salt in transgenic potato plants. Mol. Biotechnol. 2013, 54, 803-817. [CrossRef] [PubMed]

26. Sakuma, Y.; Liu, Q.; Dubouzet, J.G.; Abe, H.; Shinozaki, K.; Yamaguchi-Shinozaki, K. DNA-binding specificity of the ERF/AP2 domain of Arabidopsis DREBs, transcription factors involved in dehydration- and cold-inducible gene expression. Biochem. Biophys. Res. Commun. 2002, 290, 998-1009. [CrossRef] [PubMed]

27. Wang, Q.Y.; Guan, Y.C.; Wu, Y.R.; Chen, H.R.; Chen, F.; Chu, C.C. Overexpression of a rice OsDREB1F gene increases salt, drought, and low temperature tolerance in both Arabidopsis and rice. Plant Mol. Biol. 2008, 67, 589-602. [CrossRef] [PubMed]

28. Gilmour, S.J.; Zarka, D.G.; Stockinger, E.J.; Salazar, M.P.; Houghton, J.M.; Thomashow, M.F. Low temperature regulation of the Arabidopsis CBF family of AP2 transcriptional activators as an early step in cold induced COR gene expression. Plant J. 1998, 16, 433-442. [CrossRef] [PubMed]

29. Dubouzet, J.G.; Sakuma, Y.; Ito, Y.; Kasuga, M.; Dubouzet, E.G.; Miura, S.; Seki, M.; Shinozaki, K.; Yamaguchi-Shinozaki, K. OsDREB genes in rice, Oryza sativa L., encode transcription activators that function in drought-, high-salt- and cold-responsive gene expression. Plant J. 2003, 33, 751-763. [CrossRef] [PubMed]

30. Qin, F.; Sakuma, Y.; Li, J.; Liu, Q.; Li, Y.Q.; Shinozaki, K.; Yamaguchi-Shinozaki, K. Cloning and functional analysis of a novel DREB1/CBF transcription factor involved in cold-responsive gene expression in Zea mays L. Plant Cell Physiol. 2004, 45, 1042-1052. [CrossRef] [PubMed]

31. Shan, D.P.; Huang, J.G.; Yang, Y.T.; Guo, Y.H.; Wu, C.A.; Yang, G.D.; Gao, Z.; Zheng, C.C. Cotton GhDREB1 increaces plant tolerance to low temperature and is negatively regulated by gibberellic acid. New Phytol. 2007, 176, 70-81. [CrossRef] [PubMed] 
32. Ito, Y.; Katsura, K.; Maruyama, K.; Taji, T.; Kobayashi, M.; Seki, M.; Shinozaki, K.; Yamaguchi-Shinozaki, K. Functional analysis of rice DREB1/CBF-type transcription factors involved in cold-responsive gene expression in transgenic rice. Plant Cell Physiol. 2006, 47, 141-153. [CrossRef] [PubMed]

33. Sung, D.Y.; Kaplan, F.; Lee, K.J.; Guy, C.L. Acquired tolerance to temperature extremes. Trends Plant Sci. 2003, 8, 179-187. [CrossRef]

34. Zhang, X.; Fowler, S.G.; Cheng, H.; Lou, Y.; Rhee, S.Y.; Stockinger, E.J.; Thomashow, M.F. Freezing-sensitive tomato has a functional CBF cold response pathway, but a CBF regulation that differs from that of freezing-tolerant Arabidopsis. Plant J. 2004, 39, 905-919. [CrossRef] [PubMed]

35. Livak, K.J.; Schmittgen, T.D. Analysis of relative gene expression data using real-time quantitative PCR and the $2^{-\Delta \Delta C t}$ method. Methods 2001, 25, 402-408. [CrossRef] [PubMed]

36. Hardwick, K.; Baker, N.R. In vivo measurement of chlorophyll content of leaves. New Phytol. 1973, 72, 51-54. [CrossRef]

37. Taji, T.; Ohsumi, C.; Iuchi, S.; Seki, M.; Kasuga, M.; Kobayashi, M.; Yamaguchi-Shinozaki, K.; Shinozaki, K. Important roles of drought- and cold-inducible genes for galactinol synthase in stress tolerance in Arabidopsis thaliana. Plant J. 2002, 29, 417-426. [CrossRef] [PubMed]

38. Bates, L.S.; Waldren, R.P.; Teare, I.D. Rapid determination of free prolin for water-stress studies. Plant Soil 1973, 39, 205-207. [CrossRef]

(C) 2016 by the authors; licensee MDPI, Basel, Switzerland. This article is an open access article distributed under the terms and conditions of the Creative Commons Attribution (CC-BY) license (http://creativecommons.org/licenses/by/4.0/). 